\title{
A modified suture technique for the treatment of patients with pernicious placenta previa and placenta accreta spectrum: a case series
}

\author{
Lei Zhu ${ }^{1}$, Junli Lu ${ }^{1}$, Wenyang Huang ${ }^{1}$, Jing Zhao ${ }^{1}$, Menghui $\mathrm{Li}^{1}$, Huiyu Zhuang ${ }^{1}$, Yanfang Li ${ }^{1}$, Hao Liu ${ }^{1}$, \\ Lingyun $\mathrm{Du}^{2}$
}

${ }^{1}$ Department of Gynecology and Obstetrics, Beijing Chaoyang Hospital, Capital Medical University, Beijing, China; ${ }^{2}$ Department of Obstetrics, Tongzhou Maternal \& Child Health Hospital of Beijing, Beijing, China

Contributions: (I) Conception and design: L Zhu, J Lu; (II) Administrative support: J Lu; (III) Provision of study materials or patients: All authors; (IV) Collection and assembly of data: All authors; (V) Data analysis and interpretation: L Zhu; (VI) Manuscript writing: All authors; (VII) Final approval of manuscript: All authors.

Correspondence to: Junli Lu. Beijing Chaoyang Hospital, Capital Medical University, 8 Gongti Nanlu, Chaoyang District, Beijing 10020, China. Email: lujunli200868@126.com.

\begin{abstract}
Background: Pernicious placenta previa complicated by placenta accreta spectrum (PAS) often leads to hysterectomy or even maternal death due to massive bleeding. In recent years, the application of balloons has received increasing attention. It is easier to use and has reasonably good effect. However, for some patients, especially those who still have some placental residue, there might still be active bleeding. To solve this problem, we propose a method of pressure sutures around the balloon to provide a better hemostasis effect.

Methods: An observational study was conducted on patients with pernicious placenta previa and PAS at the Beijing Chaoyang Hospital, Beijing, China, between January 2018 and January 2021. During surgery, an intrauterine balloon was used to compress the hemorrhage site, and two or more absorbable sutures were placed around the uterus to apply strong pressure on the balloon. This method is an updated modification of the Lu-suture which uses a Foley catheter balloon and only one suture. The main improvements include choosing different kinds of balloons depending on various conditions and the addition of a suture below the balloon to provide much stronger pressure and prevent the balloon slipping out through the dilated cervix.

Results: A total of 10 women underwent the procedure. The mean estimated intraoperative blood loss was $1,190 \pm 548 \mathrm{~mL}$. Post-surgery, the blood loss was less than $200 \mathrm{~mL}$ in all patients. The mean blood transfusion [packed red blood cells (pRBC)] required was $2.2 \pm 2.6$ units. The mean hemostatic time was $8.1 \pm 3.4$ minutes. Conclusions: The modified suture technique provided an easy, cheap, and efficient surgical choice for patients with pernicious placenta previa and PAS.
\end{abstract}

Keywords: Pernicious placenta previa; placenta accreta spectrum; Lu-suture; postpartum hemorrhage; case series

Submitted Apr 10, 2021. Accepted for publication Jun 24, 2021.

doi: 10.21037/atm-21-2318

View this article at: https://dx.doi.org/10.21037/atm-21-2318

\section{Introduction}

Pernicious placenta previa is a type of placenta previa that occurs when the placenta attaches to a previous cesarean scar (1). About $50 \%$ of these patients are complicated by placenta accreta spectrum (PAS) (2). Placenta accreta spectrum, formerly known as morbidly adherent placenta, refers to the range of pathologic adherence of the placenta, including placenta accreta, placenta increta, and placenta percreta. During cesarean sections, the risk of massive bleeds is generally high due to poor contraction of the lower segments of the uterus and the residual placenta. This often results in hysterectomy or even maternal death (3). 
It can also lead to severe complications such as premature birth and neonatal blood loss (4).

Compared with normal PAS (no placenta attaches to previous cesarean scar), the incidence of placenta percreta is higher in the patients complicated with pernicious placenta previa, which leads to higher incidence of massive bleeding, hysterectomy and maternal death (4). Cesarean hysterectomy is currently considered the preferred method in most national societies. But sometimes the accuracy of imaging diagnosis of PAS is not good enough. Direct hysterectomy may lead to over treatment, complications during hysterectomy and loss of fertility (5).

A series of conservative treatments can be administered, including uterus artery ligation, external compression with different methods of uterine sutures (6-10), uterine cavity compressions using different types of balloons (11-14), placement of abdominal aortic balloons prior to surgery, or any combination of these methods (15-17). Currently, there are no standardized surgical procedures for this condition, because there are many methods, and there is no sufficient clinical data to accurately compare and evaluate the effect and prognosis of each suture method. In addition, most of the operation of these suture methods is complex, and it is difficult to promote them in clinical practice.

In recent years, the application of balloons has received increasing attention. It is easier to use and has reasonably good effect. We had reported a method called the Lu-suture , which used an absorbable suture to encircle the top of the balloon to provide additional pressure (18). The hemostasis effect has been shown to be significantly superior to that of using the balloon alone (18). However, for some patients, especially those who still have some placental residue, there might still be active bleeding. To avoid the need to perform hysterectomies in such patients, this report suggested the use of a second suture to put further pressure from the bottom of the balloon. Together with the first suture on the top, all bleeding regions should be tightly compressed by the balloon and provide a better hemostasis effect.

We present the following article in accordance with the AME Case Series reporting checklist (available at https:// dx.doi.org/10.21037/atm-21-2318).

\section{Methods}

A single center prospective consecutive observational study was conducted on patients with pernicious placenta previa and PAS at the Beijing Chaoyang Hospital, a public tertiary referral center, Beijing, China, between January 2018 and
January 2021. All patients were followed up to 42 days after delivery.

Inclusion criteria were:

(I) 20-45 years old;

(II) A history of cesarean section;

(III) Diagnosis of placenta previa by ultrasound or MRI;

(IV) Placenta attached to the uterine scar;

(V) Diagnosis of PAS by MRI.

Exclusion criteria were:

(I) Multiple pregnancy;

(II) Combination with hematological diseases;

(III) PAS ruled out during cesarean section.

Standard protocols for the management of pernicious placenta previa are in use in our institution. Magnetic resonance imaging (MRI) is performed in all patients prior to cesarean sections to select an appropriate incision site which avoids penetration of the placenta. This is crucial for avoiding large uterine bleeds before the fetus is delivered. After the fetus is delivered, the uterus and placenta are gently massaged to promote placenta detachment. If the placenta cannot be naturally detached, as much of the placental tissue as possible must be removed manually. If the patient presents with steady bleeding, a modified Lusuture will be performed. All procedures performed in this study involving human participants were in accordance with the Declaration of Helsinki (as revised in 2013). The study was approved by the ethics committee of Beijing Chaoyang Hospital (No. 2013-R-135) and informed consent was taken from all the patients.

The surgical steps are as follows:

(I) The bladder does not need to be reflected downward;

(II) A Bakri balloon or a 24 Fr Foley catheter balloon is prepared. The type of balloon applied will depends on the height of the bleeding position. A 24 Fr Foley catheter balloon can be injected with $60-120 \mathrm{~mL}$ of water and a Bakri balloon can be injected with $500 \mathrm{~mL}$ or more. The balloon is inserted by passing the distal end of the catheter through the cervix with an assistant pulling vaginally and a collection bag is set up;

(III) A size 1 Vicryl suture is used to place a stitch through both sides of the avascular zone below the origin of the round ligament from the uterus. A suture is placed on the top of the balloon. When tightening the suture, an additional downward pressure can be provided to ensure the balloon compresses the lower segment of the uterus, so as to achieve hemostasis. If the bleeding does not stop, 


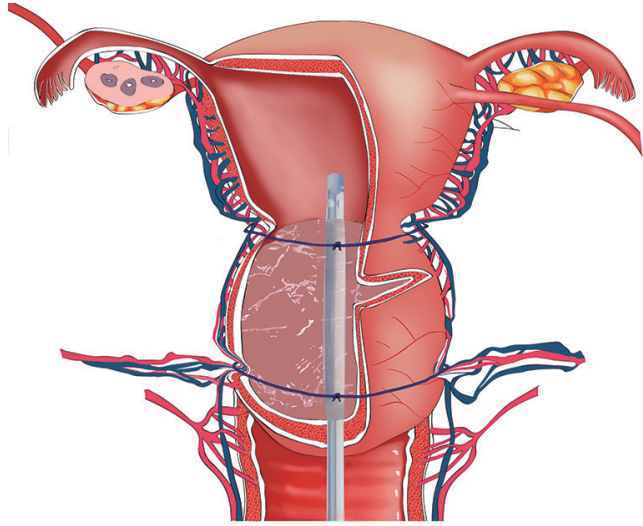

Figure 1 Schematic diagram of the modified Lu-suture. An intrauterine balloon is inserted into the uterus. A size 1 Vicryl suture is used to place a stitch through both sides of the avascular zone below the origin of round ligament from the uterus. The first suture is placed on the top of the balloon. The second suture is placed slightly below the previous suture, still within the avascular zone, but located around the bottom of the balloon.

a second suture is applied. The second suture is positioned slightly below the previous suture, still within the avascular zone, but around the bottom of the balloon. When tightening the suture, additional pressure can be applied again to ensure the balloon is compact against the bleeding site (Figures 1 and 2);

(IV) The uterine incision is then closed in the usual manner;

(V) The balloon remains in situ for 24-48 hours, according to the risk of bleeding; and

(VI) Prostaglandins will be administered before removal of the balloon. The fluid in the balloon is slowly extracted and the balloon is removed after confirmation that there is no active bleeding. If there is active bleeding, the balloon can be expanded again.

\section{Statistical analysis}

SPSS19.0 statistical software (IBM Corp., Armonk, NY) was used for processing. Mean (standard deviation) or median [interquartile range $(\mathrm{Q} 1, \mathrm{Q} 3)$ ] was used as measurement data.

\section{Results}

A total of 10 patients with pernicious placenta previa and PAS underwent treatment with the modified $\mathrm{Lu}$-suture

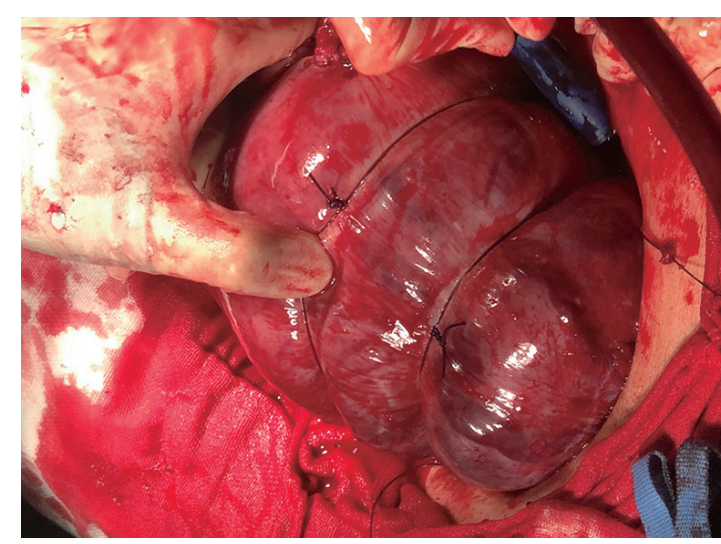

Figure 2 Use of the modified Lu-suture during surgery. To avoid penetration of the placenta, the incision was made at the fundus of the uterus. The suture presses the balloon tightly on the lower segment of the uterus.

technique. Table 1 shows the clinical data of the women included in this study. The mean estimated intraoperative blood loss was $1,190 \pm 548 \mathrm{~mL}$. Post-surgical blood loss was less than $200 \mathrm{~mL}$ in all cases. The mean blood transfusion [packed red blood cells (pRBC)] required was $2.2 \pm 2.6$ units. The mean hemostatic time was $8.1 \pm 3.4$ minutes. All infants were delivered in good condition. All patients were followed up to 42 days after delivery. All patients had good compliance and no loss follow-up occurred. None of the patients experienced complications of fever, uterine erosion, or renal dysfunction. None of the women experienced delayed hemorrhage that required re-laparotomy or hysterectomy.

\section{Discussion}

Postpartum hemorrhage still a significant cause of maternal mortality worldwide. With the increasing rates of cesarean sections, the incidence of pernicious placenta previa complicated by PAS has also increased $(19,20)$. These patients often experience uncontrollable bleeding during operations, which can lead to hysterectomies and even maternal death (21-23).

Cesarean hysterectomy is currently considered the preferred method in most national societies. However, there may be technical difficulties in some cases, such as severe pelvic pathologic adhesion and deep pelvis neovascularization with invasion to parametrium and adjacent organs. It is associated with a significant risk of maternal mortality (7\%), genitourinary injury (6-29\%), 
Table 1 Clinical data of patients managed with the modified Lu-suture

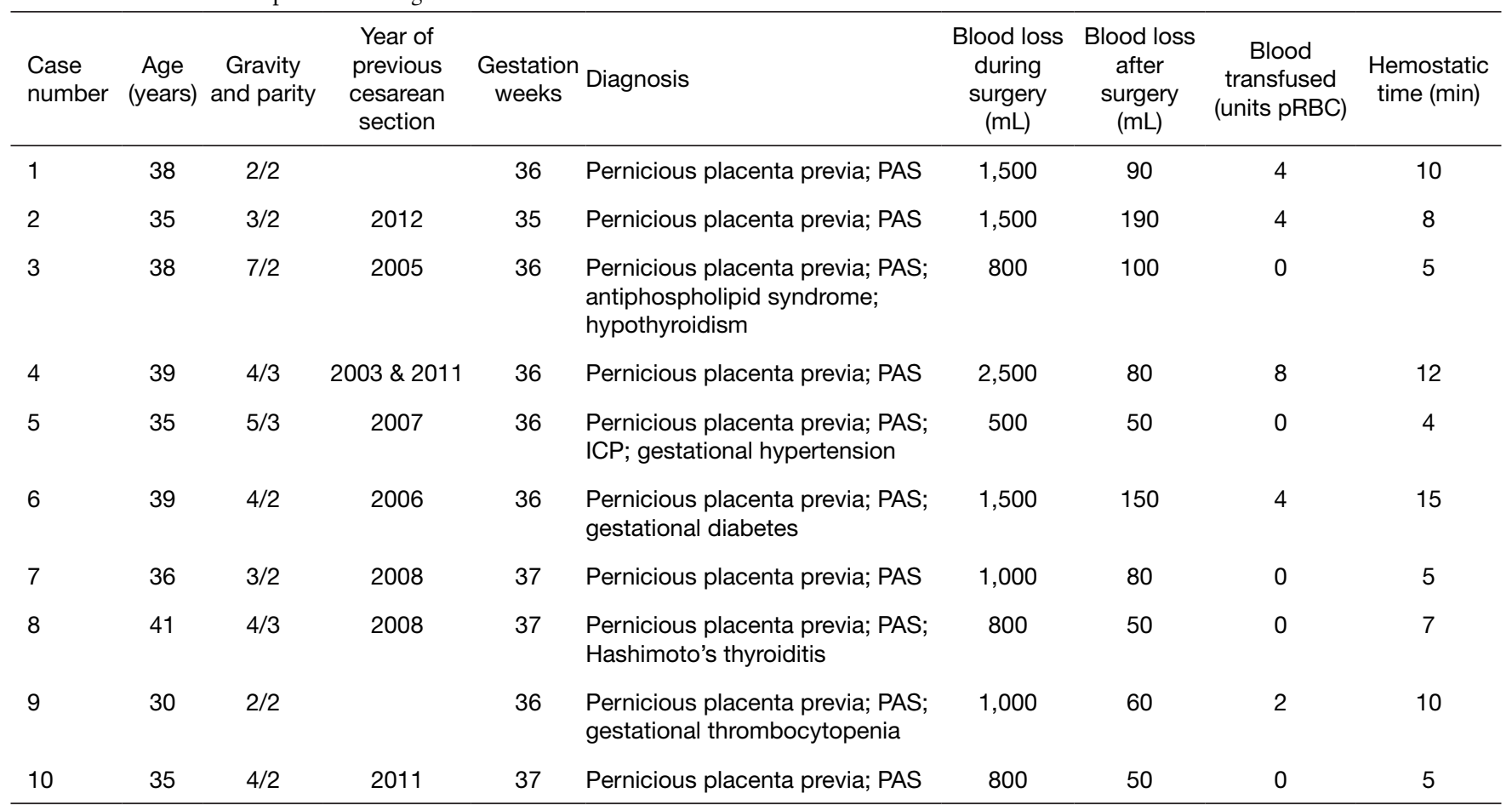

ICP, intrahepatic cholestasis of pregnancy; pRBC, packed red blood cell; PAS, placenta accreta spectrum.

massive transfusion (13\%), and further surgery (33\%) (5). There are also many conservative treatments which can be used for this situation such as all kinds of uterine sutures, intrauterine balloons, and combination of these methods. But currently, there are no standardized surgical procedures for this condition because each approach has its advantages and disadvantages. For all kinds of suture technique of lower uterine segment, the bladder is needed to be pushed down to avoid bladder and ureter injury. But in these cases, the bladder often adhered to the lower uterine segment, or the placenta implanted into the bladder, which makes the suture difficult.

To solve this problem, we proposed a new surgical procedure in our institution. Preoperative routine MRI scans are conducted for these patients. According to the position of placenta, the optimal incision is selected to avoid penetrating the placenta such that when the fetus is delivered, there will be minimal heavy bleeding. Furthermore, this will allow sufficient time to evaluate the condition of the lower uterine segment before the placenta is delivered, and the most efficient method of hemostasis can be selected.

Among the different hemostasis methods, there has been increasing focus on uterine balloons. In a previous study, we reported the use of the Lu-suture method where a balloon is used to compress the hemorrhage site, and an absorbable suture is added to provide additional pressure to the balloon (18). The hemostasis effect was found to be significantly more efficacious than using balloon compression alone. However, for patients with pernicious placenta previa complicated by PAS, especially those who still have a degree of placental residue, there may still be active bleeding even after application of the Lu-suture. In this current study, we reported an updated surgical procedure that improves upon the Lu-suture. A second additional suture is used to apply pressure on the bottom of the balloon (Figure 1). Together with the first suture on the top of the balloon, the active bleeding areas should all be tightly compressed by the balloon. If necessary, more sutures can be used around the residual placental segments until the bleeding stops. This study demonstrated that this technique can quickly and effectively achieve hemostasis, with less postoperative bleeding and no serious complications. This method was found to be suitable for patients with pernicious placenta previa and PAS as it can achieve a much more efficient hemostasis effect. 
Prior to the development of the modified Lu-suture method, for very high-risk patients, the abdominal aortic balloon would be placed before the operation to reduce the amount of intraoperative bleeding. With the application of the Lu-suture method, the abdominal aortic balloon is not necessary for these patients. Uterine bleeding can be controlled more efficiently and effectively, and the amount of blood loss is minimal. Moreover, the associated medical costs are reduced and thus, this method is suitable for widespread application. Although no cases of uterine necrosis were observed, it is important to note that the tightness of the suture should be based on hemostasis and care should be taken to avoid over-tightening the suture to reduce related complications.

It is noteworthy that all the needling points for our patients were located in the avascular zone. After the upper suture is tightened, the ascending branches of the uterine artery will be blocked and the bleeding of the uterine body is thus reduced. The lower suture does not need to be too low as the suture can be wrapped under the balloon. When it is tightened, the descending branches of the uterine artery will also be blocked. Therefore, the risk of ureter and bladder injury are minimal, and the overall difficulty of the operation is also greatly reduced. This technique has been promoted through advanced obstetricians training and academic conferences. If it can be widely used, it will greatly reduce the amount of bleeding in such patients, reduce the incidence of hysterectomy and maternal complications.

This modified Lu-suture method can also be used in other situations, such as bleeding in the lower uterine segment due to other reasons, especially when the cervix is too loose, and the balloon cannot be fixed in the lower uterine segment. This method is not suitable for all patients with PAS. For patients with large placenta percreta area, hysterectomy is not difficult to practice, and no desire to have another child, cesarean section hysterectomy may be a better choice.

\section{Conclusions}

The modified Lu-suture method is simple, safe, and costeffective. It can greatly minimize the amount of blood loss and reduce the risk of hysterectomies and maternal mortality. Therefore, it is recommended for use in the treatment of lower uterine bleeding in patients with pernicious placenta previa and PAS. Therefore, further well- designed studies with large samples are warranted to confirm our findings.

\section{Acknowledgments}

Funding: None.

\section{Footnote}

Reporting Checklist: The authors have completed the AME Case Series reporting checklist. Available at https:// dx.doi.org/10.21037/atm-21-2318

Data Sharing Statement: Available at https://dx.doi. org/10.21037/atm-21-2318

Conflicts of Interest: All authors have completed the ICMJE uniform disclosure form (available at https://dx.doi. org/10.21037/atm-21-2318). The authors have no conflicts of interest to declare.

Ethical Statement: The authors are accountable for all aspects of the work in ensuring that questions related to the accuracy or integrity of any part of the work are appropriately investigated and resolved. All procedures performed in this study involving human participants were in accordance with the Declaration of Helsinki (as revised in 2013). The study was approved by the ethics committee of Beijing Chaoyang Hospital (No. 2013-R-135) and informed consent was taken from all the patients.

Open Access Statement: This is an Open Access article distributed in accordance with the Creative Commons Attribution-NonCommercial-NoDerivs 4.0 International License (CC BY-NC-ND 4.0), which permits the noncommercial replication and distribution of the article with the strict proviso that no changes or edits are made and the original work is properly cited (including links to both the formal publication through the relevant DOI and the license). See: https://creativecommons.org/licenses/by-nc-nd/4.0/.

\section{References}

1. Kollmann M, Gaulhofer J, Lang U, et al. Placenta praevia: incidence, risk factors and outcome. J Matern Fetal Neonatal Med 2016;29:1395-8.

2. Yang T, Li N, Hou R, et al. Development and validation of a four-microRNA signature for placenta accreta spectrum: an integrated competing endogenous RNA network analysis. Ann Transl Med 2020;8:919.

3. Abuhamad AZ; ACOG Committee on Practice Bulletins- 
Obstetrics. ACOG Practice Bulletin, clinical management guidelines for obstetrician-gynecologists number 98, October 2008 (replaces Practice Bulletin number 58, December 2004). Ultrasonography in pregnancy. Obstet Gynecol 2008;112:951-61.

4. Li P, Tang Y, Jiang Y, et al. Analysis of clinical features of 231 cases with pernicious placenta previa: A retrospective cohort study. Medicine (Baltimore) 2021;100:e25023.

5. Kutuk MS, Ak M, Ozgun MT. Leaving the placenta in situ versus conservative and radical surgery in the treatment of placenta accreta spectrum disorders. Int J Gynaecol Obstet 2018;140:338-44.

6. Marasinghe JP, Condous G. Uterine compression sutures for post-partum bleeding with atony; modification of the B-Lynch suture. Aust N Z J Obstet Gynaecol 2009;49:67-70.

7. Palacios-Jaraquemada JM. Efficacy of surgical techniques to control obstetric hemorrhage: analysis of 539 cases. Acta Obstet Gynecol Scand 2011;90:1036-42.

8. Li GT, Li XF, Wu B, et al. Longitudinal parallel compression suture to control postopartum hemorrhage due to placenta previa and accrete. Taiwan J Obstet Gynecol 2016;55:193-7.

9. Zhao B, Lv M, Dong T, et al. Transverse parallel compression suture: a new suturing method for successful treating pernicious placenta previa during cesarean section. Arch Gynecol Obstet 2020;301:465-72.

10. Du X, Xie X, Wang Y. Uterine artery suture: a preventive approach for pernicious placenta previa. Cell Biochem Biophys 2014;68:407-10.

11. Albayrak M, Ozdemir I, Koc O, et al. Post-partum haemorrhage from the lower uterine segment secondary to placenta praevia/accreta: successful conservative management with Foley balloon tamponade. Aust N Z J Obstet Gynaecol 2011;51:377-80.

12. Beckmann MM, Chaplin J. Bakri balloon during cesarean delivery for placenta previa. Int J Gynaecol Obstet 2014;124:118-22.

13. Arduini M, Epicoco G, Clerici G, et al. B-Lynch suture, intrauterine balloon, and endouterine hemostatic suture for the management of postpartum hemorrhage due to placenta previa accreta. Int J Gynaecol Obstet 2010;108:191-3.

14. Kumru P, Demirci O, Erdogdu E, et al. The Bakri balloon for the management of postpartum hemorrhage in cases with placenta previa. Eur J Obstet Gynecol Reprod Biol
2013;167:167-70.

15. Luo F, Xie L, Xie P, et al. Intraoperative aortic balloon occlusion in patients with placenta previa and/or placenta accreta: a retrospective study. Taiwan J Obstet Gynecol 2017;56:147-52.

16. He Q, Li YL, Zhu MJ, et al. Prophylactic abdominal aortic balloon occlusion in patients with pernicious placenta previa during cesarean section: a systematic review and meta-analysis from randomized controlled trials. Arch Gynecol Obstet 2019;300:1131-45.

17. Wei Y, Luo J, Luo D. Comparison of Efficacy between Internal Iliac Artery and Abdominal Aorta Balloon Occlusions in Pernicious Placenta Previa Patients with Placenta Accrete. Gynecol Obstet Invest 2019;84:343-9.

18. Zhu L, Zhang Z, Wang H, et al. A modified suture technique for hemorrhage during cesarean delivery complicated by complete placenta previa. Int J Gynaecol Obstet 2015;129:26-9.

19. Imudia AN, Awonuga AO, Dbouk T, et al. Incidence, trends, risk factors, indications for, and complications associated with cesarean hysterectomy: a 17-year experience from a single institution. Arch Gynecol Obstet 2009;280:619-23.

20. Baba Y, Matsubara S, Ohkuchi A, et al. Anterior placentation as a risk factor for massive hemorrhage during cesarean section in patients with placenta previa. J Obstet Gynaecol Res 2014;40:1243-8.

21. Garmi G, Salim R. Epidemiology, etiology, diagnosis, and management of placenta accreta. Obstet Gynecol Int 2012;2012:873929.

22. Bowman ZS, Manuck TA, Eller AG, et al. Risk factors for unscheduled delivery in patients with placenta accreta. Am J Obstet Gynecol 2014;210:241.e1-6.

23. Silver RM, Landon MB, Rouse DJ, et al. Maternal morbidity associated with multiple repeat cesarean deliveries. Obstet Gynecol 2006;107:1226-32.

(English Language Editor: J. Teoh)

Cite this article as: Zhu L, Lu J, Huang W, Zhao J, Li M, Zhuang H, Li Y, Liu H, Du L. A modified suture technique for the treatment of patients with pernicious placenta previa and placenta accreta spectrum: a case series. Ann Transl Med 2021;9(14):1140. doi: 10.21037/atm-21-2318 